\title{
Prostate brachytherapy in New South Wales: patterns of care study and impact of caseload on treatment quality
}

\author{
Stephen R. Thompson, MBBS, MD, FRANZCR, PhD',2,3, Prof. Geoff P. Delaney, MBBS, FRANZCR, MD, PhD',3,4, \\ Gabriel S. Gabriel, MBBS, MD, MPH, FAFPHM!1,3, Michael A. Izard, MBBS, MD, FRANZCR, MMedicalHumb.6, \\ George Hruby, BHB, MBChB, MD, FRANZCR5,7, Raj Jagavkar, MBBS (Hons), MD, FRANZCR33,8, Joseph Bucci, MBBS, \\ FRACP, MD, FRANZCR 3,9 , Prof. Michael B. Barton, OAM, MBBS, MD, FRANZCR',3 \\ 'Collaboration for Cancer Outcomes Research and Evaluation (CCORE), Liverpool Hospital, Sydney, ${ }^{2}$ Department of Radiation Oncology, \\ Prince of Wales Hospital, Sydney. ${ }^{3}$ University of New South Wales, Faculty of Medicine, Sydney, ${ }^{4}$ University of Western Sydney, \\ Faculty of Medicine, Sydney, ${ }^{5}$ Faculty of Medicine, University of Sydney, Sydney, ${ }^{6}$ Mater Hospital Radiotherapy, North Sydney. \\ 7Sydney Cancer Centre, Sydney, ${ }^{8}$ Department of Radiation Oncology, St Vincent's Hospital, Sydney, 'Cancer Care Centre, St George Hospital, \\ Sydney, NSW, Australia
}

\begin{abstract}
Purpose: We performed the first comprehensive, population-based brachytherapy (BT) Patterns of Care Study in the Australian setting. Herein we report on prostate BT and assess the technical quality of BT practice, focusing on whether a caseload effect could be identified in New South Wales (NSW).

Material and methods: Site visits were made to all radiation oncology departments in NSW that delivered prostate BT, collecting relevant data on NSW residents treated with prostate BT in 2003. Overall quality of NSW prostate BT treatment was assessed using benchmarks including treatment of appropriate prostate cancer disease risk category, absence of (relative) physical contraindications, optimal planned and treated dosimetry, and pre-/post-implant planning/CT. Quality was compared between higher and lower caseload departments.

Results: One hundred and fifty-seven (67\%) patients underwent temporary BT and 79 (33\%) permanent seed BT. Prostate BT was concentrated in five departments, with three of four departments with active programmes treating greater than the recommended 25 cases. Rates of concordance with quality benchmarks were high (85-99\%) with no consistent caseload effect identified.

Conclusions: Prostate BT in NSW in 2003 was generally of high quality and a caseload effect on quality could not be identified. This may be because the number of departments was insufficient to determine a caseload effect, or because the prostate BT was largely concentrated in a small number of high caseload departments.
\end{abstract}

Key words: brachytherapy, caseload, patterns of care study, prostate cancer, quality, radiotherapy.

\section{Purpose}

Retrospective patterns of care studies (POCS) seek to improve the quality of technical care by examining processes, structures, and/or outcomes of care. The rationale is that differences exist in care, that these differences can be measured against explicit criteria and against each other, that deficiencies in care may affect outcome, and that these deficiencies, having been identified, are correctable, thereby improving patient outcome [1,2]. This is the first comprehensive, population-based brachytherapy (BT) POCS in the Australian setting. Collection of these data enabled an overview of actual NSW BT use for comparison with our model of optimal BT utilization [3]. A second aim was to assess the technical quality of BT practice by comparing with known quality benchmarks. Herein we focus on these results as they pertain to prostate BT quality.

\section{Material and methods}

A retrospective BT POCS for NSW residents treated in 2003 was performed in 2005. Site visits were made to all radiation oncology departments in NSW that delivered BT. Data were collected by the primary author and entered into an access database and then transferred into SPSS v15.0. Logic checks and frequency analyses were performed on the data to identify errors and inconsistencies.

\footnotetext{
Address for correspondence: Stephen R. Thompson, MBBS, MD, FRANZCR, PhD, Department of Radiation ～Received: 19.03 .2014 Oncology, Prince of Wales Hospital, Australia, High St, 2031 Randwick, Australia, phone: +61 29382 2501, Accepted: 18.09 .2014 fax: +61 29382 2550, « e-mail: stephen.thompson@sesiahs.health.nsw.gov.au

Published: 30.12 .2014
} 
Overall quality of NSW prostate BT treatment was assessed using near-contemporaneous published benchmarks drawn from evidence-based peer-reviewed guidelines, including: treatment of appropriate prostate cancer disease risk category [4-9], absence of (relative) physical contraindications to BT [4-6,10], optimal planned and treated dosimetry [5], and pre/post-implant planning/ CT $[4,5]$. Quality was compared between higher and lower caseload departments. These were defined as: the two departments that performed more permanent prostate seed BT (SBT) compared with the two departments that performed fewer (institution numbers 2 and 5, versus 1 and 3); and the two departments that performed more temporary high dose rate (HDR)/ pulsed dose rate (PDR) iridium-192 brachytherapy (TBT) were compared to the two departments that performed fewer (institution numbers 3 and 5, versus 2 and 4).

Categorical variables were compared using Pearson $X^{2}$ test, with data entered as counts and analysed as proportions. Ordering of variables was addressed by testing for Linear Trend. Means of two variables were compared using two-sample $t$-test (two-tailed), and variance was tested using Levene's test for homogeneity of variance.

All institutions and patients were de-identified. Permission for the study was obtained from all relevant Radiation Oncology Departmental Directors and Human Research Ethics Committees. The study was carried out in accordance with the Helsinki Declaration.

\section{Results}

In NSW in 2003, 260 patients were treated with prostate BT $(47 \%$ of all NSW BT) at five institutions. After exclusion of 24 patients resident interstate, 236 NSW prostate cancer patients remained as the cohort of this study undergoing BT. Temporary brachyhterapy was administered to $157(67 \%)$ patients and $79(33 \%)$ received SBT. Institutional caseload is shown in Table 1 . The two higher caseload SBT institutions treated 32 and 37 SBT patients, compared to 1 and 9 patients in the two lower caseload SBT institutions. The two higher caseload TBT institutions treated 47 and 80 TBT patients, the two lower caseload TBT institutions treated 14 and 16 patients.
One department had a temporarily inactive prostate BT programme due to resource constraints and only treated one patient. Of the other four departments, three treated more than the previous UK recommended minimum of 25 cases (SBT + TBT) [11,12].

Mean patient age was 67 years. Prostate TBT patients were older than SBT patients (mean 69 vs. 64 years, $p<0.001$ ), with a greater proportion being greater than 65 years $(75 \%$ vs. $44 \%, p<0.001)$. Prostate cancer T stage, Gleason score and PSA are combined to create low risk (LR), intermediate risk (IR) and high risk (HR) disease categories, as per the NCCN recommendations [6]. Prostate cancer treatment by institution and risk category is shown in Table 1.

Seed brachytherapy monotherapy is contraindicated in HR disease [4-8], this criterion was met in 99\% of SBT patients. Temporary brachyhterapy (with EBRT) is indicated for patients with non-metastatic disease $[6,9]$, with this criterion being achieved in $98 \%$ of patients. There was no significant difference between departments by caseload.

Relative physical contraindications to prostate BT are: very large prostates (> 40-60 cc); significant lower urinary tract symptoms (LUTS), with International Prostate Symptom Score (IPSS) cut-off being $0-9,0-15$, or $0-19$; and prior trans-urethral resection of prostate (TURP) $[4-6,10]$. Temporary brachyhterapy patients were more likely to have larger prostates than SBT patients: (37\% vs. $26 \%$ $>40 \mathrm{cc}$, including $9 \%$ vs. $0 \%>60 \mathrm{cc}, p=0.03$ ). For SBT, there was no difference by caseload, whereas patients in lower caseload TBT departments were more likely to have large prostates $(14 \%$ vs. $7 \%>60 \mathrm{cc}, p=0.012)$. Data on pre-treatment IPSS were available for 93 (39\%) patients. Mean IPSS was 4.2 (range 0-16). Only one patient scored greater than 15 (score 16). There was no difference in average IPSS or proportions of patients having mild (IPSS 0-9) or moderate (IPSS 10-19) LUTS by BT type $(p=0.63)$ or by caseload amongst SBT patients $(p=0.68)$, but more TBT patients at lower caseload departments had moderate LUTS $(20 \%$ vs. $3 \%, p=0.048)$. Six percent of SBT patients had prior TURP compared to $15 \%$ of TBT patients $(p=0.048)$, but there was no association with caseload.

Table 1. Prostate brachytherapy by institution and prostate cancer risk category

\begin{tabular}{|c|c|c|c|c|c|c|c|c|c|}
\hline \multirow[t]{3}{*}{ Institution } & \multicolumn{4}{|c|}{ Seed BT } & \multicolumn{5}{|c|}{ Temporary BT* } \\
\hline & \multicolumn{3}{|c|}{ Risk category } & \multirow[t]{2}{*}{ Total } & \multicolumn{4}{|c|}{ Risk category } & \multirow[t]{2}{*}{ Total } \\
\hline & LR & $\mathrm{IR}$ & $\mathrm{HR}$ & & LR & IR & HR & Mets. & \\
\hline 1 & 1 & 0 & 0 & 1 & - & - & - & - & - \\
\hline 2 & 26 & 5 & 1 & 32 & 0 & 12 & 4 & 0 & 16 \\
\hline 3 & $9^{*}$ & 0 & 0 & 9 & 4 & 20 & 22 & 1 & 47 \\
\hline 4 & - & - & - & - & 0 & 10 & 4 & 0 & 14 \\
\hline 5 & 22 & 15 & 0 & 37 & 2 & 36 & 40 & 2 & 80 \\
\hline Total & 58 & 20 & 1 & 79 & 6 & 78 & 70 & 3 & 157 \\
\hline
\end{tabular}

$B T$ - brachytherapy, $L R$ - low risk, $I R$ - intermediate risk, $H R$ - high risk, Mets. - metastases *Two SBT and all TBT patients had combined modality treatment with EBRT 
Table 2. Permanent seed brachytherapy - technical factors

\begin{tabular}{lccc} 
Institution & $\begin{array}{c}\text { Number of seeds } \\
\text { Mean (range) }\end{array}$ & $\begin{array}{c}\text { Prostate volume } \\
\text { Mean (range), cc }\end{array}$ & Organs at risk \\
\hline 1 & $62(\mathrm{n} / \mathrm{a})$ & $23(\mathrm{n} / \mathrm{a})$ & Urethra, rectum \\
\hline 2 & $100(78-118)$ & $36(24-51)$ & Urethra \\
\hline 3 & $80(62-92)$ & $21(15-28)$ & Urethra, rectum, bladder, seminal vesicle \\
\hline 5 & $77(48-105)$ & $34(15-55)$ & Urethra, rectum
\end{tabular}

n/a-not applicable

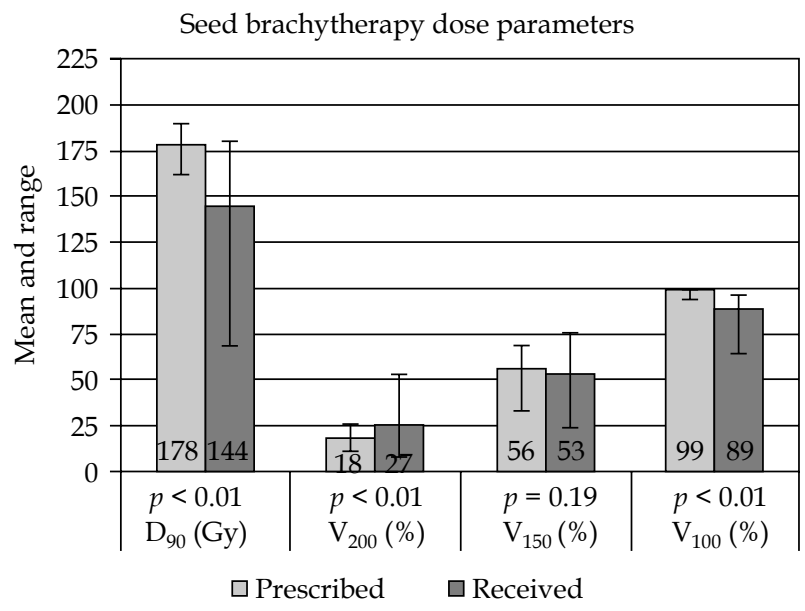

Fig. 1. Permanent seed brachytherapy. Prescribed and received dose parameters. Column graph indicates mean and whiskers indicate range, $\mathrm{y}$-axis reprepresents Gray for $\mathrm{D}_{90}$ and $\%$ for $\mathrm{V}_{200 / 150 / 100}$

\section{Permanent iodine-125 seed brachytherapy}

In all cases, planning was conducted as a day-stay procedure, 145 Gy was prescribed to the covering 100\% isodose using iodine- 125 seeds, and insertion was then under general anaesthetic as day-only or overnight. Other implant descriptors are tabulated (Table 2).

Post-implant CT to calculate dosimetry is recommended for all patients [4,5] and was performed on $69(87 \%)$ of 79 patients with no difference by caseload. Prescribed and received doses are shown in Figure 1. The data indicate that prescribed dosimetry was superior in terms of prostate coverage and dose homogeneity compared to that actually achieved. Optimal implant is one, in which $\mathrm{D}_{90}$ (dose in Gy covering $>90 \%$ of target) $>140$ Gy [5], this was prescribed in all cases and $71 \%$ received at least this dose, with no difference by caseload. Mean prescribed $\mathrm{D}_{90}$ was greater in lower SBT caseload departments (185 Gy vs. $177 \mathrm{~Gy}, p<0.001)$ with less variation $(p=0.01)$ and prescribed $\mathrm{V}_{150}(\%$ target volume covered by $150 \%$ of prescribed dose) was greater in lower SBT departments ( $62 \%$ vs. $56 \%$ of prostate, $p=0.01$ ) with less variation $(p=0.01)$. There was no significant difference by caseload in received $D_{90}$ and $V_{150}$ and in prescribed/received $V_{200}$ and $\mathrm{V}_{100}$.

\section{Temporary Iridium-192 high-dose-rate/ pulsed-dose-rate brachytherapy}

Treatment protocols varied between departments and are detailed in Table 3, and the boxplot in Figure 2 shows dose parameters prescribed.

There was greater variability in $\mathrm{D}_{90}$ and $\mathrm{V}_{100}$ in lower caseload compared to higher caseload institutions ( $p=0.017$ and $p=0.001$, respectively) with no difference in their means. Mean $\mathrm{V}_{150}$ and $\mathrm{V}_{200}$ were significantly lower in higher caseload departments $(27 \%$ vs. $36 \%$, $p<0.001$ and $9 \%$ vs. $15 \%, p<0.001)$. By extrapolation from SBT data [5], optimal $D_{90}$ is believed to be at least as great as the prescribed dose. This was achieved in $86 \%$ of cases (90\% vs. $71 \%$, higher / lower caseload, $p=0.039)$.

Table 3. Temporary brachytherapy - technical factors

\begin{tabular}{|c|c|c|c|c|c|c|c|c|}
\hline Institution & $\begin{array}{l}\text { Treatment } \\
\text { order }\end{array}$ & $\begin{array}{c}\text { EBRT } \\
\text { prescription } \\
\text { (median) }\end{array}$ & $\begin{array}{c}\text { BT } \\
\text { prescription } \\
\text { (median) }\end{array}$ & $\begin{array}{l}\text { Dose } \\
\text { rate }\end{array}$ & PTV & $\begin{array}{l}\text { Catheter; } \\
\text { median } \\
\text { (range) }\end{array}$ & $\begin{array}{l}\text { Prostate } \\
\text { volume; } \\
\text { mean } \\
\text { (range), cc }\end{array}$ & Organs at risk \\
\hline 2 & $\begin{array}{c}\text { BT then } \\
\text { EBRT }\end{array}$ & 45 Gy, 25\# & 19.5 Gy, $3^{\#}$ & PDR & $\begin{array}{l}\text { CTV }+5 \mathrm{~mm} \\
\text { sup/inf }\end{array}$ & $18(17-20)$ & $48(28-88)$ & Urethra, rectum \\
\hline 3 & $\begin{array}{c}\text { BT then } \\
\text { EBRT }\end{array}$ & 50.4 Gy, $28^{\#}$ & $18 \mathrm{~Gy}, 3^{\#}$ & PDR & $\begin{array}{c}\text { CTV }+5 \mathrm{~mm} \\
\text { ant/lat, } \\
+1 \mathrm{~mm} \text { post }\end{array}$ & $17(17-17)$ & $47(20-85)$ & Urethra, rectum \\
\hline 4 & $\begin{array}{l}\text { Usually BT } \\
\text { then EBRT }\end{array}$ & 46 Gy, 23\# & $19.5 \mathrm{~Gy}, 3^{\#}$ & HDR & $\begin{array}{l}\text { Prostate + } \\
\text { extension }\end{array}$ & $19(13-26)$ & $42(26-79)$ & Urethra, rectum \\
\hline 5 & $\begin{array}{c}\text { BT then } \\
\text { EBRT }\end{array}$ & 50.4 Gy, $28^{\#}$ & $18 \mathrm{~Gy}, 3^{\#}$ & HDR & Prostate & $20(18-20)$ & $27(14-47)$ & Urethra, rectum \\
\hline
\end{tabular}

BT - brachytherapy, EBRT - external beam radiotherapy, Gy - Gray, ${ }^{*}$ - fractions, PDR - pulsed dose rate, HDR - high dose rate, PTV - planning target volume, CTV-clinical target volume 


\section{Discussion}

Brachytherapy for the treatment of prostate cancer has a well-established role [13]. In NSW in 2003, 67\% of prostate BT patients were treated with TBT, always as a boost with EBRT, and almost always for patients with IR-HR disease. Thirty-three percent were treated with SBT, almost all monotherapy, generally for patients with LR disease. This is markedly different to the American experience, with the USA POCS for 1999 revealing that only $10 \%$ of BT treatments were TBT $(69 \%$ combined with EBRT and $31 \%$ monotherapy) and $90 \%$ were permanent SBT [14]. This difference in the type of BT used between Australia and USA is likely to be explained by differences in funding arrangements, which has had the practical result that in Australia permanent SBT is almost entirely limited to monotherapy of privately insured patients with LR disease, and TBT is generally used as a boost to EBRT to treat both public and private patients with IR-HR disease [3].

Prostate BT in NSW generally corresponded to guideline recommendations, with benchmarks achieved in 85$99 \%$ of cases. Seed brachytherapy (monotherapy) is contraindicated in HR disease [4-8], and TBT (with EBRT) is contraindicated for patients with metastatic disease $[6,9]$. These criteria were met in $>98 \%$ of patients. This is similar to the American experience: in the 1999, prostate BT POCS only $1.1 \%$ of SBT (monotherapy) patients had HR disease [14]. Few NSW patients were treated in the presence of relative physical contra-indications (prior TURP, large prostate size, and significant LUTS). Temporary brachyhterapy patients were more likely than SBT patients to have large prostates or to have undergone prior TURP. This difference probably reflects technical differences between the two modalities and the fact that the evidence that TBT patients have greater toxicity in the presence of these contraindications is weaker and more recent than in the setting of SBT [4-6] with only one guideline listing these factors as a contra-indication to TBT [10].

New South Wales SBT practice was in line with the American [5] and European [4] technical recommendations. All NSW patients appropriately underwent preplanning, and 145 Gy was prescribed to the covering $100 \%$ isodose in all cases. Post-implant CT was performed for 69 of 79 patients (86\%) in NSW, compared to only $47 \%$ of patients in the 1999 US POCS [14]. In NSW, $\mathrm{D}_{90} \geq 140$ Gy was achieved to $71 \%$ of patients. This compares favourably with published series from large institutions: $43 \%$ at Leeds [15] and 58\% at Memorial Sloan Kettering Cancer Center [16]. New South Wales TBT boost practice also corresponded well to guideline technical recommendations [10]. Although no dose response data have been determined from the literature, there is a commonly used range of doses that have been shown to be efficacious: 45-54 Gy EBRT and 12-20 Gy in 2-4 fractions TBT boost [10]. All but two patients were treated within this dose range, and the NSW departments routinely assessed dose to organs at risk.

An important issue for prostate BT is whether BT caseload affects BT quality. This has been shown to be the case for cervical cancer brachytherapy [17]. The Royal College of Radiologists of the UK have previously recommended
TBT dose parameters, whole cohort

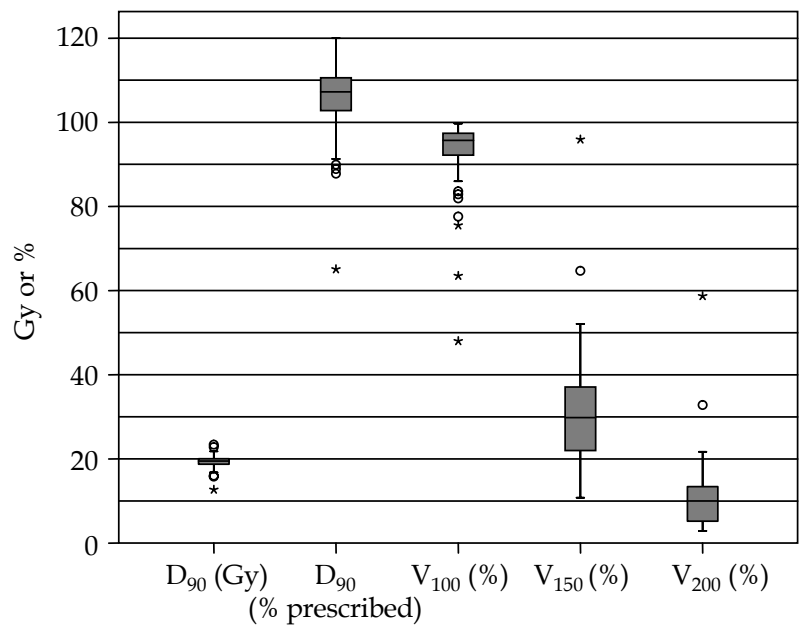

Fig. 2. Temporary brachytherapy. Dose parameters. Horizontal line - median value, vertical box $-25^{\text {th }}-75^{\text {th }}$ percentiles, vertical line - range. The y-axis represents Gray for $\mathrm{D}_{90}(\mathrm{~Gy}), \%$ of prescribed dose for $\mathrm{D}_{90}$ (\% prescribed) and

$\%$ of prostate volume for $\mathrm{V}_{200 / 150 / 100}$

a minimum of 25 prostate BT cases be treated per centre per year, and a minimum of 5 cases per physician per year, although no studies were referenced to support these recommendations [11]. In NSW, prostate BT was highly concentrated: of 15 NSW radiation oncology departments, only five delivered prostate BT. Prostate BT workload per centre in NSW was comparable to that of the UK and greater than continental Europe (Table 4). Three of four NSW departments with active prostate BT programmes in 2003 treated more than the UK recommended minimum.

In the case of permanent SBT, there were no differences by caseload with regard to likelihood of treating in the presence of physical contra-indications to BT, use of post-implant $\mathrm{CT}$, or prescribed/delivered $\mathrm{D}_{90}$, with only minor differences in other dosimetric parameters. For patients treated with TBT, there were some differences in likelihood of treating according to guideline recommendations by caseload: patients in lower TBT caseload departments were more likely to have large prostates $>60 \mathrm{cc}$ ( $14 \%$ vs. $7 \%$ ), but the limited data regarding greater risk of urinary toxicity with large prostate volumes in TBT patients emerged only after 2003 [10,22-25], and therefore the applicability of using this measure as a quality benchmark for the 2003 NSW POCS is questionable. Patients in the lower caseload TBT departments were more likely to have moderate LUTS as measured by IPSS ( $20 \%$ vs. $3 \%)$, although no patients in either type of department had severe symptoms, this being the group at greatest risk of urinary retention after BT. There was no difference in prior TURP by caseload. Although there were some differences in dosimetry by caseload (with less variability in $D_{90}$ and $V_{100}$ and greater likelihood of $D_{90}$ being greater than the prescribed dose), there are no dose response data, and therefore no dose parameters that define an optimal TBT implant [10].

Thus, despite a broad literature showing the existence of a learning curve and an effect of provider volume 
Table 4. New South Wales prostate brachyhterapy workload per centre and comparison with other jurisdictions.

NB: One NSW department with temporarily resource constrained BT programme treating only 1 patient is excluded from Table; if included, NSW figures/centre would be: mean: 47.2 , range: $1-117,60 \% \geq 25$ cases

\begin{tabular}{|c|c|c|c|c|c|c|c|}
\hline $\begin{array}{l}\text { Cases/Centre/ } \\
\text { Year }\end{array}$ & NSW 2003 & ABS 1992 [18] & $\begin{array}{c}\text { Western Europe } \\
2002[19]\end{array}$ & UK 1998 [20] & UK 2004 [11] & $\begin{array}{c}\text { Italy } 2002 \\
{[21]} \\
\end{array}$ & $\begin{array}{c}\text { Spain } 2002 \\
{[21]} \\
\end{array}$ \\
\hline Mean & 59 & - & 35.3 & 30 & 53.6 & 6.0 & 16.3 \\
\hline Range & $14-117$ & - & - & $4-80$ & $3-177$ & $\begin{array}{l}95 \% \mathrm{Cl}: \\
1.4-10.7\end{array}$ & $\begin{array}{l}95 \% \mathrm{Cl}: \\
5.3-27.3 \\
\end{array}$ \\
\hline Other & $\begin{array}{c}75 \% \geq 25 \\
\text { cases }\end{array}$ & $\begin{array}{c}45 \%>10 \\
\text { cases }\end{array}$ & - & $\begin{array}{c}50 \% \geq 10 \\
\text { cases }\end{array}$ & $\begin{array}{c}63 \% \geq 25 \\
\text { cases }\end{array}$ & - & - \\
\hline
\end{tabular}

NSW - New South Wales, ABS - American Brachytherapy Society, UK - United Kingdom, 95\% CI - 95\% confidence interval

on outcomes following prostate BT, ranging from first 10-20 cases, but with an effect detectable out to to hundreds [26-31], we were unable to consistently demonstrate this effect. There may be a number of reasons why the current study did not find a strong caseload effect on indicators of prostate BT quality, despite good evidence in the literature for this effect. There is a lack of robust dosimetry quality benchmarks for TBT, although the detected greater variability in $D_{90}$ and $V_{100}$ at the lower caseload departments may be clinically significant. Although the sample included 236 prostate BT patients, these were treated in only five departments by six brachytherapists, and therefore the number of institutions is probably too small to detect a difference. One interpretation may be that the high overall quality of prostate BT in NSW may indeed confirm a caseload effect, with three of the four active departments achieving the previous UK benchmark rate of $\geq 25$ cases/ year. In these four departments, all prostate brachytherapists were treating well above the UK benchmark rate of $>5$ cases/year. By 2003, all the NSW brachytherapists at the four active departments had performed many more than the standard learning curve caseload of 10-20. Even at the lower caseload departments, numbers appeared small due to resource constraints or the BT programmes having only just commenced, but the brachytherapists themselves were generally experienced. It may be that for quality, overall brachytherapist experience and having passed beyond the learning curve is more important than absolute numbers in any one calendar year.

This study did have a number of limitations. It was retrospective, and represents state-of-play in 2003, and therefore not necessarily reflecting current NSW practice. Nevertheless, no data have yet emerged implying that new technologies have rendered brachytherapist experience/technical skill inconsequential, and therefore we believe that these data assessing caseload and quality remain relevant. The study included only five departments. Robust TBT treatment quality indicators against, which performance can be measured are lacking. Importantly, this study reports technical factors, but not clinical outcome data for both disease control and toxicity. These are areas in which future research is likely to be beneficial.

\section{Conclusions}

In NSW in 2003 prostate BT, as measured by concordance with guideline recommendations and dosimetry parameters, was of high quality. No consistent provider caseload effect on treatment quality was found. These results may be because prostate BT was concentrated in a small number of departments, with generally high departmental caseload.

\section{Disclosure}

Authors report no conflict of interest.

This manuscript is based on data presented at the following Scientific Meetings: The Royal Australian and New Zealand College of Radiologists $56^{\text {th }}$ Annual Scientific Meeting, Sydney, October 2005; Australasian Brachytherapy Group 18 ${ }^{\text {th }}$ Annual Scientific Meeting, Brisbane, March 2009; The Royal Australian and New Zealand College of Radiologists $61^{\text {st }}$ Annual Scientific Meeting, Perth, October 2010.

\section{References}

1. Thompson S, Delaney G, Gabriel G et al. NSW brachytherapy patterns of care study: Impact of caseload on treatment quality. J Med Imag Radiat Oncol 2010; 54: A82.

2. Coia L, Hanks G. Quality Assessment in the USA: How the Patterns of Care Study Has Made a Difference. Semin Radiat Oncol 1997; 7: 146-156.

3. Thompson SR, Barton M. Optimal utilization of permanent seed brachytherapy for the treatment of prostate cancer. 2009. Submitted to NSW Department of Health. Ref Type: Report.

4. Ash D, Flynn A, Battermann J et al. ESTRO/EAU/EORTC recommendations on permanent seed implantation for localized prostate cancer. Radiother Oncol 2000; 57: 315-321.

5. Nag S, Beyer D, Friedland J et al. American Brachytherapy Society (ABS) recommendations for transperineal permanent brachytherapy of prostate cancer. Int J Radiat Oncol Biol Phys 1999; 44: 789-799.

6. National Comprehensive Cancer Network. NCCN Clinical Practice Guidelines in Oncology - v.2. 2014 - Prostate Cancer. Available at: http://www.nccn.org/professionals/physician_gls/PDF/prostate.pdf. 2014. 28-7-2014.

7. Cancer Care Ontario Genitourinary Cancer Disease Site Group. Low-dose rate brachytherapy for patients with lowor intermediate-risk prostate cancer: guideline recommendations. Available at: https://www.cancercare.on.ca/common/ pages/UserFile.aspx?fileId=254196. 2012. 28-7-2014.

8. BC Cancer Agency. Cancer Management Guidelines - Prostate. Available at: http://www.bccancer.bc.ca/HPI/CancerManage mentGuidelines/Genitourinary/Prostate/Management/.

9. Hsu IC, Yamada Y, Vigneault E et al. American Brachytherapy Society Prostate High-Dose Rate Task Group. Available 
at: http:// www.americanbrachytherapy.org/guidelines/HDRTaskGroup. pdf. 2008. 9-8-2012.

10. Kovacs G, Potter R, Loch T et al. GEC/ESTRO-EAU recommendations on temporary brachytherapy using stepping sources for localised prostate cancer. Radiother Oncol 2005; 74: $137-148$

11. Board of the Faculty of Clinical Oncology, The Royal College of Radiologists. The Role and Development of Brachytherapy Services in United Kingdom. Available at: http://www. rcr.ac.uk. 2007. (Note: the document is no longer accessible, but is available from the primary author).

12. Board of the Faculty of Clinical Oncology, The Royal College of Radiologists. The role and development of afterloading brachytherapy services in the United Kingdom. Available at: http://www.rcr.ac.uk/docs/oncology/pdf/BFCO(12)3_ brachy.pdf.2012. 28-7-2014.

13. Skowronek J. Brachytherapy in the therapy of prostate cancer - an interesting choice. Contemp Oncol 2013; 17: 407-412.

14. Lee WR, Moughan J, Owen JB et al. The 1999 patterns of care study of radiotherapy in localized prostate cancer. Cancer 2003; 98: 1987-1994.

15. Ash D, Al-Qaisieh B, Bottomley D et al. The correlation between D90 and outcome for I-125 seed implant monotherapy for localised prostate cancer. Radiother Oncol 2006; 79: 185-189.

16. Potters L, Cao Y, Calugaru E et al. A comprehensive review of CT-based dosimetry parameters and biochemical control in patients treated with permanent prostate brachytherapy. Int J Radiat Oncol Biol Phys 2001; 50: 605-614.

17. Thompson SR, Delaney GP, Gabriel GS et al. Patterns of care study of brachytherapy in New South wales: cervical cancer treatment quality depends on caseload. J Contemp Brachytherapy 2014; 6: 28-32

18. Nag S, Owen JB, Farnan N et al. Survey of brachytherapy practice in the United States: A report of the clinical research committee of the American Endocurietherapy Society. Int J Radiat Oncol Biol Phys 1995; 31: 103-107.

19. Hoskin PJ, Venselaar J. Prostate brachytherapy in Europe: Growth, practice and guidelines. Radiother Oncol 2007; 83: $1-2$.

20. Board of the Faculty of Clinical Oncology, The Royal College of Radiologists. The Role and Development of Brachytherapy Services in United Kingdom. Available at: http://www. rcr.ac.uk.2001. 15-6-2004. (Note: the document is no longer accessible but is available from the primary author).

21. Guedea F, Ventura M, Marchetti C et al. Patterns of care for brachytherapy in Europe, in Spain and Italy: comparative results. Tumori 2007; 93: 439-444.

22. Pellizzon ACA, Salvajoli JV, Maia MAC et al. Late urinary morbidity with high dose prostate brachytherapy as a boost to conventional external beam radiation therapy for local and locally advanced prostate cancer. J Urol 2004; 171: 1105-1108.

23. Deger S, Boehmer D, Roigas J et al. High dose rate (HDR) brachytherapy with conformal radiation therapy for localized prostate cancer. Eur Urol 2005; 47: 441-448.

24. Soumarova R, Homola L, Stursa M et al. Acute toxicity of conformal high dose interstitial brachytherapy boost in prostate cancer. Neoplasma 2006; 53: 410-417.

25. Skowronek J. Low-dose-rate or high-dose-rate brahcytherapy in treatment of prostate cancer - between options. J Contemp Brachytherapy 2013; 5: 33-41.

26. Stock RG, Stone NN, Tabert A et al. A dose-response study for I-125 prostate implants. Int J Radiat Oncol Biol Phys 1998; 41: 101-108.

27. Lee WR, DeGuzman AF, Bare RL et al. Postimplant analysis of transperineal interstitial permanent prostate brachytherapy: evidence for a learning curve in the first year at a single institution. Int J Radiat Oncol Biol Phys 2000; 46: 83-88.
28. Keyes M, Schellenberg D, Moravan V et al. Decline in urinary retention incidence in 805 patients after prostate brachytherapy: the effect of learning curve? Int J Radiat Oncol Biol Phys 2006; 64: 825-834.

29. Merrick GS, Grimm P, Sylvester J et al. Initial analysis of ProQura: A multi-institutional database of prostate brachytherapy dosimetry. Brachytherapy 2007; 6: 9-15.

30. Loiselle CR, Waheed M, Sylvester J et al. Analysis of the ProQura database: rectal dose, implant quality, and brachytherapist's experience. Brachytherapy 2009; 8: 34-39.

31. Chen AB, D'Amico AV, Neville BA et al. Provider case volume and outcomes following prostate brachytherapy. J Urol 2009; 181: 113-118. 